\title{
Glaucoma Detection Using Artificial Neural Network
}

\author{
Sheeba O., Jithin George, Rajin P. K., Nisha Thomas, and Sherin George
}

\begin{abstract}
Glaucoma is the term applied to a group of eye diseases that gradually result in loss of vision by permanently damaging the optic nerve, the nerve that transmits visual images to the brain. Here the detection of glaucoma is done by image processing. The screening of patients for the development of glaucoma potentially reduces the risk of blindness in these patients by $\mathbf{5 0 \%}$. Here neural network is trained to recognize the parameters for the detection of different stages of the disease. The neuron model has been developed using feed forward back propagation network. Here the program is developed using Matlab. The images acquired using medical imaging techniques are analysed in Matlab. Matlab provide variety of options for image processing that enable us to extract the required features and information from the images. The software can be used to detect the early stages of glaucoma.
\end{abstract}

Index Terms-Glaucoma, image processing, Matlab, neural network.

\section{INTRODUCTION}

Glaucoma is characterized by a particular pattern of progressive damage to the optic nerve that generally begins with a subtle loss of side vision (peripheral vision). An elevation in the pressure within the eye (the intraocular pressure, or IOP) is generally, but not always, associated with the development of glaucoma, although additional factors are also likely to play a role in its development. In some cases, glaucoma may occur in the presence of normal eye pressure. This form of glaucoma is believed to be caused by poor regulation of blood flow to the optic nerve. Glaucoma is the diagnosis given to a group of ocular conditions that contribute to the loss of retinal nerve fibres with a corresponding loss of vision. Glaucoma therefore is a disease of the optic nerve, the nerve bundle which carries images from the eyes retina to the brain.

Glaucoma usually affects both eyes, but one eye may be more severely affected than the other. As the total amount of fluid within the eye increases, so does the pressure, similar to over inflating a tire. Glaucoma is said to be one of the leading causes of blindness in people over the age of 40. Loss of peripheral vision is the earliest symptom. Left untreated the field of vision will continue to narrow leading to tunnel vision. If detected early, loss of vision can most often be prevented.

Manuscript received March 1, 2013; revised July 24, 2013.

Sheeba O is with Department of Electronics \& Communication, T.K.M. College of Engineering, Kerala, India(e-mail: shb.odattil@gmail.com)

Jithin George was with Robert BOSCH multinational engineering and electronics (automotive) company, headquartered in Gerlingen, near Stuttgart.

Rajin P.K is with Tata Consultancy Services Ltd, a reputed multinational IT services and outsourcing firm.

Nisha Thomas and Sherin George are with Tata Consultancy Services Ltd, a reputed multinational IT services and outsourcing firm.

\section{SYMPTOMS AND CLASSIFICATION}

The first and foremost variation due to glaucoma is in the intraocular pressure of eye. In fact glaucoma is said to be a disease due to elevation in eye pressure [1]. The usual value of intraocular pressure varies between 10 to $20 \mathrm{mmHg}$. But in patients with glaucoma it may increase (but not always). Due to this pressure increase the nerve fibres begin to die. When these fibres die the light that falls on these regions will not induce any sense of vision. Thus the spot becomes blind. In fact the blind spot is called cup. Due to glaucoma the cup area may increase, correspondingly the disc area reduces. It is a slow process that it may take years for a small change. As a result of these changes the side vision of the patient reduces gradually [2].

The increase in blind area increases the cup size assuming the disc size to remain constant. The vertical cup to disc ratio of the eye is defined as the ratio of the diameter of the cup to diameter of the optic disc.

Cup to disc ratio $=\frac{\text { Diameter of the cup }}{\text { Diameter of the disc }}$

Due to glaucoma the cup enlarges so the cup to disc ratio increases in patients with glaucoma. The cup may not increase uniformly to all directions. This may create irregularities in the thickness of Inferior (I), Superior(S), Nasal (N), and Temporal (T).Another parameter that can be taken into consideration is the area of neuro retinal rim (NRR). Neuro retinal rim is the region between the cup and the disc. As the cup enlarges due to glaucoma the area of the NRR gets reduced. As the loss of nerve fibre becomes more severe the peripheral vision of the patient reduces. The different stages of the disease are classified as mild, normal and severe.

\section{PRocessing OF IMAGeS}

The images are obtained using fundus camera and are processed in Matlab. A number of morphological operations are used for this purpose. First the images are cropped interactively to select the required disc-cup area. All further processes are done on the cropped image. Lighter objects on the edge are suppressed and the resulting image is then converted into grey scale. On the resulting grey scale image morphological operations are performed.

Mathematical morphology is an approach to image analysis based on set theory [3]. Here two fundamental morphological operations, dilation and erosion are used in terms of intersection of an image with a translated shape for extracting features from an image. Dilation is an operation that "grows" objects in an image. A shape referred to as a structuring element controls the extent of growing. The 
erosion of a set by a structuring element is the set of pixel positions for which a structuring element placed with its reference point that will be contained completely within the set. An opening is similar to erosion, except that it consists of all points of the structuring element when the structuring element can be placed within a set. Dilation is an erosion of the background of a set. Erosion "shrinks" objects in an image. Dilation and erosion can be used in various combinations. In morphological opening, erosion removes small objects and the subsequent dilation tends to restore the shape of the objects that remain. The structuring element used is a disk of size 200 .

The morphologically opened output is treated as the background image and is subtracted from the grey scale image obtained [4]. The intensity of the resulting image is adjusted and converted into binary image by thresholding representing the cup disc region. The areas of cup, disk and NRR are found from the extracted binary regions.

\section{ARtificial NeURAL Networks}

An artificial neural network is an information processing system that has certain performance characteristics in common with biological neural networks [5]. A neural network is characterized by its pattern of connections between the neurons, its method of determining the weights on the connections and its activation function. A neural net consists of a large number of simple processing elements called neurons or nodes. Each neuron is connected to other neurons by means of directed communication links, each with an associated weight. The weights represent information being used by the net to solve a problem. Each neuron has an internal state called its activation or activity level, which is a function of the inputs it has received. A neuron sends its activation as a signal to several other neurons.

Artificial neural networks consist of many nodes, processing units analogous to neurons in the brain. The neural net can be a single layer or multilayer net. In a single layer net there is a single layer of weighted interconnections. A multi-layer artificial neural network comprises an input layer, output layer and hidden (intermediate) layer of neurons The activity of neurons in the input layer is represents the raw information that is fed into the network. The activity of neurons in the hidden layer is determined by the activity of input neurons and the connecting weights between the input and hidden units. Similarly the behavior of the output units depends on the activity of the neurons in the hidden layer and the connecting weights between hidden and the output layers [5]. A neural network can be trained to perform a particular function by adjusting the values of the connections (weights) between elements.

Commonly neural networks are adjusted, or trained, so that a particular input leads to a specific target output. There, the network is adjusted, based on a comparison of the output and the target, until the network output matches the target. Typically many such input/target pairs are needed to train a network.

\section{BACK PROPAGATION}

Back propagation is the generalization of the Widrow-Hoff learning rule to multiple-layer networks and nonlinear differentiable transfer functions. Input vectors and the corresponding target vectors are used to train a network until it can approximate a function, associate input vectors with specific output vectors, or classify input vectors in an appropriate way as defined by you. Networks with biases, a sigmoid layer, and a linear output layer are capable of approximating any function with a finite number of discontinuities.

Standard back propagation [6] is a gradient descent algorithm, as is the Widrow-Hoff learning rule, in which the network weights are moved along the negative of the gradient of the performance function. The term back propagation refers to the manner in which the gradient is computed for nonlinear multilayer networks. There are a number of variations on the basic algorithm that are based on other standard optimization techniques, such as conjugate gradient and Newton methods

Properly trained back propagation networks tend to give reasonable answers when presented with inputs that they have never seen. Typically, a new input leads to an output similar to the correct output for input vectors used in training that are similar to the new input being presented. This generalization property makes it possible to train a network on a representative set of input/target pairs and get good results without training the network on all possible input/output pairs [7].

The simplest implementation of back propagation learning updates the network weights and biases in the direction in which the performance function decreases most rapidly, the negative of the gradient [8].

\section{TRAINING THE NETWORK}

Once the network weights and biases are initialized, the network is ready for training. The training process requires a set of examples of proper network behaviour network inputs and target outputs. During training the weights and biases of the network are iteratively adjusted to minimize the network performance function. The default performance function for feed forward networks is mean square error (mse), the average squared error between the networks outputs and the target outputs. All these algorithms use the gradient of the performance function to determine how to adjust the weights to minimize performance [9]. The gradient is determined using back propagation, which involves performing computations backward through the network.

There are generally four steps in the training process:

1) Assemble the training data.

2) Create the network object.

3) Train the network.

4) Simulate the network response to new inputs.

\section{Methodology}

The parameters used are Intra Ocular Pressure, Central Corneal Thickness, Nerve Fiber Layer Thickness and Cup to Disc Ratio. The Nerve Fiber Layer Thickness of Nasal Region, Temporal Region, Inferior Region and Superior Region are given as parameters. Matlab neural network toolbox provides a complete set of functions and a 
graphical user interface (GUI) for the design and simulation of neural networks. GUI can be used for creating, training and simulating neural networks. Fig.1 shows the display window for the training process.

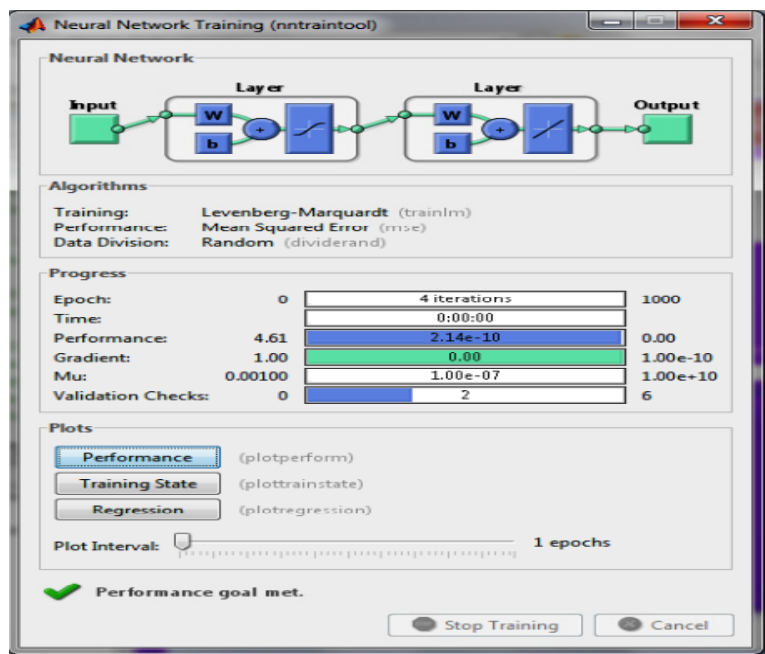

Fig. 1. Display window showing the training process

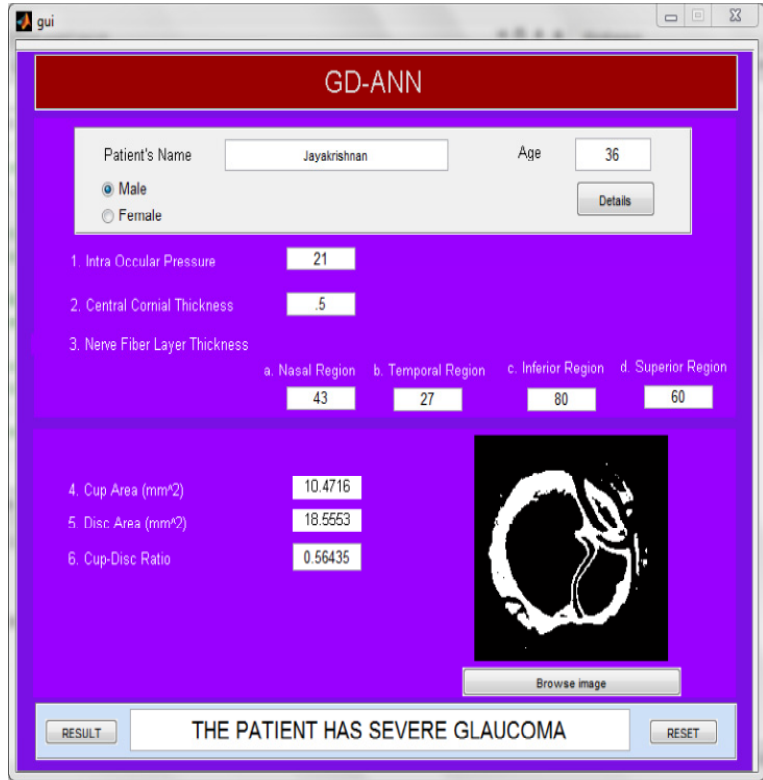

Fig. 2. Display window showing the result of testing

A two layer back propagation network was employed for the classification of the disease. The tranefer function used was $\log$ sigmoid transfer function. The network was trained using parameters of 20 patients.. The training data was used to teach the network to classify the disease as normal, mild or severe. Testing was done with parameters of 28 patients with glaucoma and 12 normal subjects including trained ones. Out of these 34 were identified correctly, Fig. 2 shows the result of testing with parameters of one patient.

\section{CONCLUSION}

Glaucoma is actually originating due to the increased pressure within eye ball leading to the damage of optic nerve. Here Matlab is used for training and simulating artificial neural network to detect the presence of glaucoma and classify the disease as mild, severe and normal.. The various parameters are easily extracted using Matlab and compared with standard values using neural network

The artificial neural network makes the Glaucoma detection accurate and adaptive. The advantage of the system is simplicity of operation. The manual segmentation is extremely difficult and moreover the reproducibility is low. This software intended to help the doctors in their decision making process. To make this more user friendly graphical user interface is also given which makes the handling of this tool very simple. In future application, it can be used to detect more eye diseases by taking more parameters.

\section{ACKNOWLEDGMENT}

The authors would like to thank Dr. Mahesh G. Retinal Surgeon and Dr. Savitha Bhat, MS, DO, DNB, MNAMS, Glaucoma sr-consultant, Giridhar Eye Institute, Kochi for their valuable suggestions. Retinal images are courtesy of Giridhar Eye Institute, Cochin.

\section{REFERENCES}

[1] J. F. Cullen, "The pale optic disc: Some observations of a europeanneuro-ophthalmologist in south-east asia," Asian Journal of Opthalmology, vol. 2, no. 3, 2000.

[2] P. Karyampudi, L. Wang, P. P. Chen, and R. P. Mills, "Measurement of large optic discs using the nerve fibre analyser, GDx" in Proceedings of the XIIIth International Perimetric Society Meeting, Gardone Riviera (BS), Italy, September 6-9, 1998, pp. 395-402.

[3] O. Sheeba and A. Sukeshkumar, "Automated diagnosis of macular degeneration through image processing," Journal of The Institution of Engineers, India, pp. 3-6, 2008.

[4] O. Sheeba and A. Sukeshkumar, "Diagnosis of eye diseases using medical imaging," Proceedings of International conference on Modelling and Simulation (MS'07), India, Calcutta University, 2007, pp. 196-200.

[5] O. Sheeba and A. Sukeshkumar, "Neural Networks in the diagnosis of Diabetic Retinopathy," International conference on Modelling and Simulation (MS'09), Trivandrum, Kerala, 2009, pp. 256-259.

[6] B. Yegnanarayana, Artificial neural networks, Prentice- Hall of India private limited, 1999.

[7] C. M. Bishop, Neural Networks for Pattern Recognition, New York: Oxford University, 1995.

[8] R. C. Gonzalez, R. E .Woods, Digital Image Processing, 2nd ed. Prentice Hall, 2002

[9] S. Haykin., Neural networks a comprehensive foundation, Pearson Education Asia, 2001.

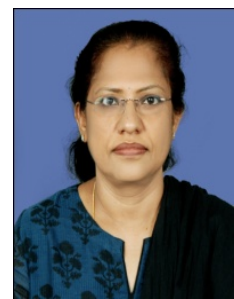

O. Sheeba was born in Thrissur, Kerala, India in October 20 1963. She received her B.Tech degree in Electronics and Communication Engineering from T.K.M College of Engineering, Kerala, India in 1987. She received her M.Tech degree From Cochin University of Science \& Technology, Cochin, Kerala. She received her $\mathrm{Ph}, \mathrm{D}$ degree from Kerala University, Kerala, India.

She is working as Professor in the department of Electronics \& Communication, T.K.M. College of Engineering, Kollam, Kerala, India.

Dr. O. Sheeba is a member of The Indian Society of Technical Education and also member of the Institution of Engineers, India

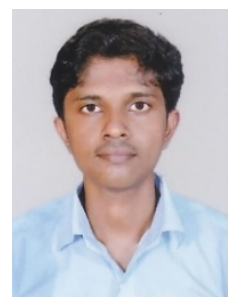

Jithin George was born in Ernakulam, Kerala in 1991. He received his B.Tech degree in Electronics and Communication Engineering from T.K.M College of Engineering, University of Kerala, India in 2012.

He has worked as an associate engineering Robert $\mathrm{BOSCH}$, a German multinational engineering and electronics (automotive) company. At present, he is doing a course on 'Quantum Mechanics and Quantum Computation’ offered by Berkeley, University of California through EDX. 


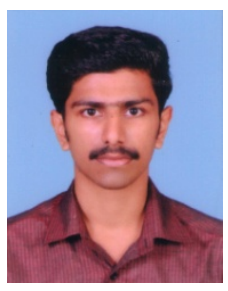

Rajin P. K. was born in Kannur, Kerala, India in 1988. He completed his B.Tech degree in Electronics and Communication Engineering from T.K.M College of Engineering, Kerala, India in 2012.

$\mathrm{He}$ is currently employed as assistant system engineer in Tata Consultancy Services Ltd, a reputed multinational IT services and outsourcing firm headquartered in Mumbai, India and he is presently working in a Business Intelligence Project.

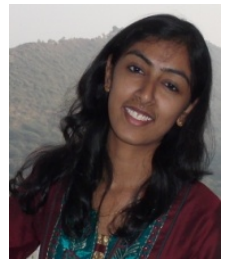

Nisha Thomas was born in Alleppey, Kerala in 1990 She received her B.Tech degree in Electronics and Communication Engineering from T.K.M College of Engineering, Kerala, India in 2012.

She is presently working as an assistant system engineer in Tata Consultancy Services Ltd, an IT services firm under the business conglomerate TATA group headquartered in Mumbai, India. Currently she is doing a migration project in mainframe technology

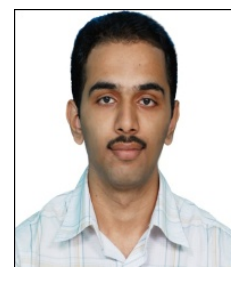

Sherin George was born in Kottayam, Kerala in 1989. He received his B.Tech degree in Electronics and Communication Engineering from T.K.M College of Engineering, Kerala, India in 2012. He is presently working as an assistant system engineer in Tata Consultancy Services Ltd, an IT services firm under the business conglomerate TATA group headquartered in Mumbai, India and he is presently working in a mainframe Project. 